\title{
Association of endothelial and red blood cell microparticles with acute myocardial infarction in Chinese: a retrospective study
}

\author{
Yujuan Yuan ${ }^{1}$, Miribani Maitusong $^{2}$, Nijiati Muyesai $^{2}$ \\ ${ }^{1}$ Department of Cardiology, ${ }^{2}$ Emergency Department, People's Hospital of Xinjiang Uygur Autonomous Region, Urumqi, China \\ Contributions: (I) Conception and design: All authors; (II) Administrative support: All authors; (III) Provision of study materials or patients: Y Yuan, N \\ Muyesai; (IV) Collection and assembly of data: M Maitusong; (V) Data analysis and interpretation: Y Yuan, N Muyesai; (VI) Manuscript writing: All \\ authors; (VII) Final approval of manuscript: All authors. \\ Correspondence to: Nijiati Muyesai. People's Hospital of Xinjiang Uygur Autonomous Region, 120 Longquan Street, Urumqi 830001, Xinjiang, China. \\ Email: mrbn831@163.com.
}

\begin{abstract}
Background: Mounting evidence suggests that endothelial cell-derived microparticles (EMPs) and red blood cell-derived microparticles (RMPs), which have procoagulant and vasoconstriction effects, are involved in the development of vascular acute cardiovascular events. The aim of this study was to analyze the circulating levels of EMPs and RMPs in patients with acute myocardial infarction (AMI), and to explore the correlations between EMPs and RMPs and the severity of coronary artery disease.

Methods: Plasma samples from 110 patients with AMI and 57 non-coronary artery disease group (nonCAD) were collected in the present study. The flow cytometry was used to measure the EMPs (CD31) and RMPs (CD235a) qualitatively and quantitatively.
\end{abstract}

Results: The levels of EMPs and RMPs in the AMI group were higher than that in the Non-CAD group, yet no significant difference was found between STEMI and non-STEMI subjects. The levels of EMPs and RMPs in multi-vessel were higher than in the single-vessel 1 disease. In the Thrombolysis in Myocardial Infarction (TIMI) risk assessment, the levels of EMPs in the high-risk group were higher than that in both intermediate- and low-risk group. The low-risk group had the lowest EMP levels, the difference between the groups being statistically significant $(\mathrm{P}=0.001)$. No significant difference in RMP levels was noted upon TIMI stratification. According to the ROC curve analysis, the areas under the curve (AUC) of EMPs and RMPs were 0.706 and 0.668 , respectively.

Conclusions: The circulating levels of EMPs and RMPs in patients with AMI are elevated, and the level of EMPs is related to the degree of coronary artery disease and the prognosis risk. The EMPs are more likely to be potential biomarkers than RMPs to provide diagnostic value for AMI.

Keywords: Acute myocardial infarction (AMI); endothelial cell-derived microparticles (EMPs); red blood cellderived microparticles (RMPs); degree of coronary artery disease; Thrombolysis in Myocardial Infarction score (TIMI score)

Submitted Feb 13, 2020. Accepted for publication Jun 04, 2020.

doi: $10.21037 /$ apm-20-397

View this article at: http://dx.doi.org/10.21037/apm-20-397

\section{Introduction}

The total number of deaths from ischaemic heart disease increased from 7.3 million in 2007 to 8.93 million in 2017, which is leading cause of death and disability worldwide (1). More than $80 \%$ of sudden cardiac death events are caused by atherosclerotic coronary artery disease $(2,3)$.
Acute myocardial infarction (AMI) occurs predominantly as a result of acute atherosclerotic plaque rupture in the coronary artery, accounting for $80 \%$ of AMI cases (4), which may result in activation of the clotting cascade with subsequent thrombus formation and occlusion of the artery. The mechanisms of arterial thrombosis are still unresolved, 
which are important because of their clinical implications.

Microparticles (MPs) are cell membrane phosphatidylserine ranging from 0.1 to $1.0 \mu \mathrm{m}$, which are containing information like mRNA, microRNAs (miRNAs), receptor and specific proteins of parent cell (5-7). MPs are expressed by cells during cell activation and apoptosis. Circulating MPs are involving in the atherosclerotic process, including chronic inflammation, and are also involving in intracoronary thrombosis and AMI (8-11).

Endothelial cell (EC) in activation and apoptosis release endothelial microparticles (EMPs). EMPs are found in the plasma of healthy individuals, but are severely increased in individuals with diabetic mellitus and coronary artery disease including atherosclerosis. EMPs can affect endothelial function through autocrine effects (10). Circulating EMPs in patients with acute coronary syndrome (ACS) impair endothelial dependence and NO-mediated vasodilation by activating the mechanisms of angiotensin II and NADPH (nicotinamide adenine dinucleotide phosphate) oxidase, thus accelerating endothelial senescence and thrombosis (12). Therefore, high concentrations of EMPs may cause vascular damage and aggravate endothelial dysfunction. Accordingly, EMPs are involving in the formation, development and rupture of atherosclerotic plaques, leading to the occurrence of acute cardiovascular events, which have certain clinical value in the diagnosis of cardiovascular diseases.

Red blood cells form MPs through exocytosis after encountering cell membrane oxidation or injury. Leroyer et al. found atherosclerotic plaques after extracting MPs from the carotid plaques and plasma of carotid endarterectomy patients, suggesting that small vessel ruptures had occurred within the plaques (13).

The aims of this study were to analyse the levels of EMPs and RMPs in AMI patients, to explore the correlation between EMPs, RMPs, coronary lesion counts and TIMI (Thrombolysis in Myocardial Infarction) risk stratification, and to determine the associations of MPs and AMI, meanwhile, to further investigate the efficiency of EMPs and RMPs as potential biomarkers of AMI.

We present the following article in accordance with the STARD reporting checklist (available at http://dx.doi. org/10.21037/apm-20-397).

\section{Methods}

\section{Study population}

The trial was conducted in accordance with the Declaration of Helsinki (as revised in 2013). The study was approved by the Ethics Committee of People's Hospital of Xinjiang Uygur Autonomous Region (No. 2017041), and all patients provided a signed informed consent form.

We recruited 110 patients with AMI and 57 patients with non-CAD in the present study from January 2015 to January 2018. The study population was selected from the inpatient Department of Cardiology at the People's Hospital of Xinjiang Uygur Autonomous Region.

The diagnostic criteria were based on previous studies as follows: (I) patients with AMI who experienced chest pain for more than 20 min, with an ECG showing elevation or depression of ST segments in at least two adjacent leads within 24 hours of onset, as well as cardiac troponin $\mathrm{T}(\mathrm{cTnT})>0.1 \mu \mathrm{g} / \mathrm{L}$ (normal value $<0.05 \mu \mathrm{g} / \mathrm{L}$ ); (II) the STEMI patients need to emergency PCI, whose chest pain were within 12 hours; (III) patients had chest pain, ECG changes and normal troponin, whose coronary angiography results were normal, who were considered non-coronary artery disease (non-CAD).

The exclusion criteria were as follows: (I) serious liver or kidney dysfunction; (II) cancer or other debilitating disease; (III) diseases of the haematopoietic system; (IV) uncontrolled infection; and (V) infarction in another location of the body, such as cerebral infarction or pulmonary embolism (VI) coronary artery spasm.

\section{Isolation of MPs}

To avoid coronary angiography and its associated interventional effects on peripheral blood MPs levels, $2 \mathrm{~mL}$ circulation blood was collected from all patients prior to coronary angiography and MP levels were subsequently detected.

Blood samples were centrifuged at 3,500 $\mathrm{g}$ at room temperature for $10 \mathrm{~min}$ to separate the bleeding cells, and the supernatant was platelet-rich plasma (PRP). PRP centrifuged a large number of platelets after centrifugation at $2,000 \mathrm{~g}$ and $10{ }^{\circ} \mathrm{C}$ for $10 \mathrm{~min}$, the supernatant was platelet-poor plasma (PPP), which followed by three centrifugations at $23,000 \mathrm{~g}$ at $4{ }^{\circ} \mathrm{C}$ for $45 \mathrm{~min}$. The supernatant was discarded, and the precipitate was plateletfree (PFP). Subsequently, $200 \mu \mathrm{L}$ of phosphate-buffered saline (PBS) was added, and the samples were mixed for $5 \mathrm{~min}$. Each sample was labelled with the date of collection and the patient admission number, which then immediately was stored at $-80^{\circ} \mathrm{C}$ for subsequent analysis of MPs.

\section{Transmission electron microscopy (TEM)}

TEM performed the MPs of that obtained by centrifugation 


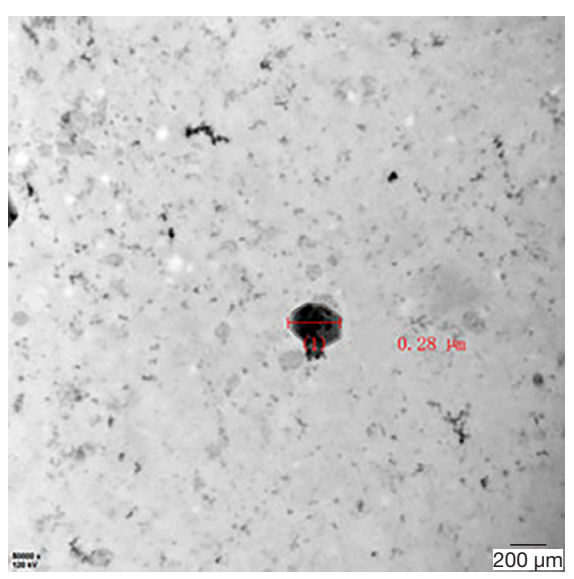

Figure 1 TEM show the structure and size of MPs. TEM, transmission electron microscopy; MPs, microparticles.

(Figure 1).

\section{Quantitation of EMPs and RMPs by flow cytometry}

The PFP was rapidly dissolved at room temperature. Next, $2.5 \mu \mathrm{L}$ of sample, $100 \mu \mathrm{L}$ of the $\mathrm{Ca}^{2+}$ vector $\mathrm{A} 23187,100 \mu \mathrm{L}$ of binding buffer and $5 \mu \mathrm{L}$ of Annexin $\mathrm{V}$ were thoroughly mixed together at room temperature for $20 \mathrm{~min}$. Subsequently, $2.5 \mu \mathrm{L}$ of endothelial cell-specific monoclonal antibody (CD31) and red blood cell-specific monoclonal antibody (CD235a) were added, followed by thorough mixing at room temperature for 20 min. Finally, $175 \mu \mathrm{L}$ of PBS was added. FACSAria II ${ }^{\mathrm{TM}}$ analysis was performed. The number of cells in the portal was 10,000 wh each time, reading at a flow rate of $35 \mu \mathrm{L} / \mathrm{min}$ for 30 seconds, counting the number of EMPs and RMPs and analyzing the fluorescence percentage of ECs and red blood cells labeled with specific monoclonal antibodies to further characterize EMPs and RMPs. The final peripheral EC- and red blood cell-derived MPs are expressed as percentages.

\section{Statistical analysis}

Continuous variables are presented as the mean \pm standard deviation $(\mathrm{SD})$ or the median \pm inter-quartile range (IQR) and were compared with Student's $t$-test or the MannWhitney $U$ test (if not normally distributed). Fisher's exact test was used for categorical data.

\section{Results}

(I) AMI patients participated in the current study and were further divided into 2 subgroups based on the type of AMI. The baseline characteristics of the patients including in this study were shown in Table 1 according to AMI and non-CAD. The most striking differences between the two groups were observed in the mean (TC), low-density lipoprotein cholesterol (LDL), creatinine clearance and left ventricular ejection fraction (LVEF). No significant differences were noted between the groups in other characteristics, such as age, sex, body mass index (BMI), hypertension, diabetes and smoking.

(II) AMI patients showed significantly higher levels of EMPs and RMPs than non-CAD patients. There were no significant differences in the EMPs and RMPs levels between STEMI and non-STEMI patients (Table 2 and Figure 2).

(III) According to the coronary angiography results, AMI patients were divided into a single-vessel disease group and a multivessel disease group. The circulating levels of EMPs and RMPs in patients with multivessel disease were higher than those in the single-vessel disease group $(\mathrm{P}<0.05)$ (Table 3).

The TIMI risk score is a clinical risk score for determining the prognosis of patients with ACS. The TIMI risk scores of the AMI patients are evaluated and divided into low-, mediumand high-risk groups. Pairwise comparison was conducted using the variance test. The EMPs levels in the three groups showed statistically significant differences $(\mathrm{P}<0.05)$, with the highest EMPs levels in the high-risk group and the lowest EMP levels in the low-risk group, while the RMP levels were not statistically significant, as shown in Table 3.

(IV) To further investigate the efficiency of EMPs and RMPs as potential biomarkers of AMI, we performed ROC curve analysis between patients with $\mathrm{AMI}$ and no-CAD. According to the outcome of ROC curve analysis, we found that the areas under the curve (AUC) of EMPs and RMPs were 0.706 and 0.668 (Figure 3).

\section{Discussion}

Atherosclerosis is the basis of coronary heart disease, and thrombosis due to atherosclerotic plaques is the main cause of acute and serious clinical complications of atherosclerotic disease (14).

MPs deliver biological messages to target cells and 
Table 1 The clinical characteristics of the non-CAD and AMI groups

\begin{tabular}{lccc}
\hline XXXXX & Non-CAD, $n=57$ & AMI, $n=110$ & Non-CAD vs. AMI, P value \\
\hline Age (years) & $59 \pm 12$ & $58 \pm 11$ & 0.080 \\
Male (n) & 39 & 85 & 0.263 \\
BMI & $26.43 \pm 3.94$ & $26.00 \pm 3.38$ & 0.484 \\
Hypertension (n) & 29 & 43 & 0.187 \\
Diabetes (n) & 8 & 23 & 0.304 \\
Smoking (n) & 14 & 25 & 0.848 \\
TC (mmol/L) & $3.39[1.40,4.64]$ & $4.13[3.51,5.06]$ & 0.000 \\
LDL (mmol/L) & $2.03 \pm 1.20$ & $2.64 \pm 0.98$ & 0.001 \\
Creatinine clearance (\%) & $96.44 \pm 34.89$ & $112.38 \pm 35.80$ & 0.026 \\
LVEF (\%) & $60.00[58.00,63.00]$ & $51.00[48.00,58.00]$ & 0.000 \\
\hline
\end{tabular}

CAD, coronary artery disease; AMI, acute myocardial infarction; BMI, body mass index; TC, total cholesterol; LDL, low-density lipoprotein cholesterol; LVEF, left ventricular ejection fraction; vs, versus.

Table 2 EMPs and RMPs levels in the AMI and non-CAD groups

\begin{tabular}{lccccc}
\hline XXXX & EMPs $\%$ & T value & P value & RMPs \% & T value \\
\hline Non-CAD & $32.16 \pm 20.10$ & 3.408 & 0.001 & $24.02 \pm 17.86$ & 4.600 \\
AMI & $43.87 \pm 21.53$ & & & $38.03 \pm 19.06$ & 0.000 \\
STEMI & $41.21 \pm 21.23$ & -1.511 & 0.134 & $36.37 \pm 16.69$ & -1.509 \\
Non-STEMI & $47.44 \pm 21.76$ & & & $40.25 \pm 21.84$ & 0.292 \\
\hline
\end{tabular}

CAD, coronary artery disease; EMP, endothelial cell-derived microparticle; RMP, red blood cell-derived microparticle; AMI, acute myocardial infarction; CAD, coronary artery disease.

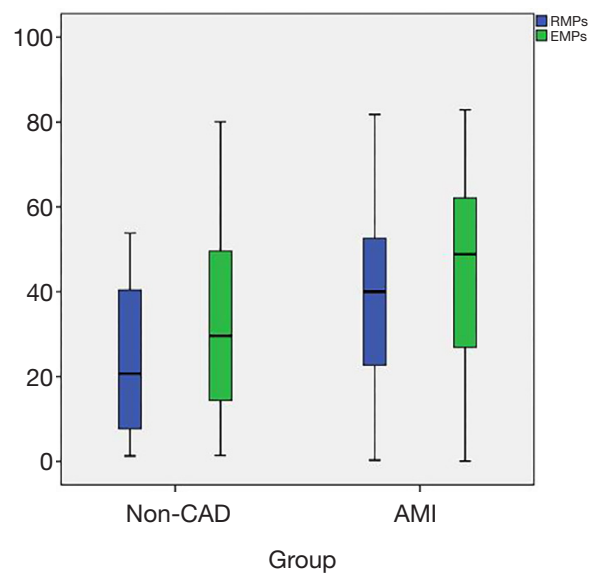

Figure 2 The levels of EMPs and RMPs in the ACS and nonCAD patients. EMP, endothelial cell-derived microparticle; RMP, red blood cell-derived microparticle; ACS, acute coronary syndrome; CAD, coronary artery disease. regulate EC dysfunction, inflammation (15), oxidative stress (16), angiogenesis (17), and permeability (18), processes that are intricately implicated in the pathogenesis of CAD and its sequelae.

Compared to control patients, procoagulant EMPs are found in greater circulatory concentrations in ACS patients and may contribute to intracoronary thrombus generation (19). EMPs release is triggered by various stimuli, which is followed by activation of various pathways that can collectively advance the progression of atherogenesis. Most importantly, oxidative stress, endogenous molecules (TNF- $\alpha$, PDGF, and interleukin) and apoptosis induce EMPs are released to undergo functional changes in blood vessels, eventually leading to the pathology of atherosclerosis.

Recent studies have shown that RMPs are released into the circulating blood due to worsening thrombosis conditions and can be detected in patients with STEMI. 
Table 3 EMPs and RMPs levels in the AMI and non-CAD groups

\begin{tabular}{|c|c|c|c|c|c|c|}
\hline$X X X X$ & EMPs \% & $\mathrm{F}$ value & $P$ value & RMPs \% & $\mathrm{F}$ value & $P$ value \\
\hline Single-vessel & $35.23 \pm 22.17$ & -2.585 & 0.011 & $31.26 \pm 20.25$ & -2.270 & 0.025 \\
\hline Multivessel & $46.97 \pm 20.57$ & & & $40.45 \pm 18.14$ & & \\
\hline \multicolumn{7}{|l|}{ TIMI } \\
\hline Intermediate-risk & $45.98 \pm 19.98$ & & & $38.91 \pm 19.23$ & & \\
\hline High-risk & $52.56 \pm 18.03$ & & & $39.78 \pm 20.03$ & & \\
\hline
\end{tabular}

EMP, endothelial cell-derived microparticle; RMP, red blood cell-derived microparticle; AMI, acute myocardial infarction; CAD, coronary artery disease; TIMI, Thrombolysis in Myocardial Infarction.

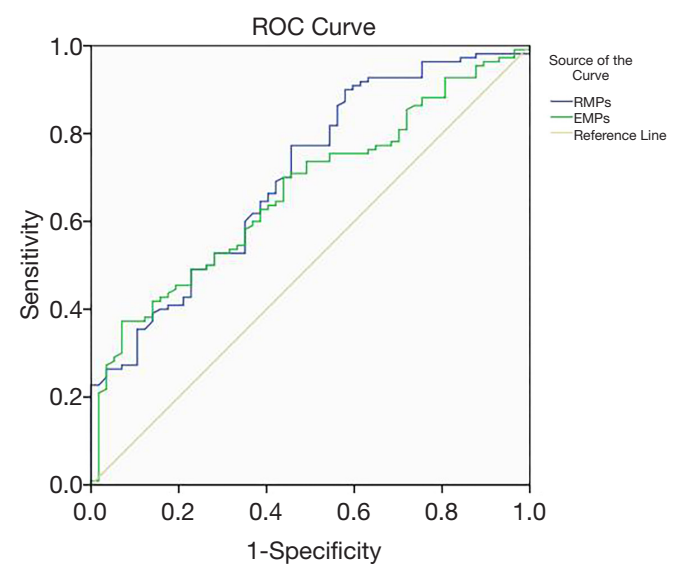

Figure 3 ROC curve analyses of EMPs and RMPs in AMI patients. EMP, endothelial cell-derived microparticle; RMP, red blood cell-derived microparticle; AMI, acute myocardial infarction.

Thus, RMPs are considered as a new marker of persistent thrombosis (20). One study found that CD235a RMP levels in the peripheral blood of STEMI patients were higher than the control group (11).

The results of our study can be summarized as follows: (I) our results suggest that the levels of EMPs and RMPs were higher in patients with $\mathrm{AMI}$ the non-CAD population, which was consistent with our previous findings (21-23); (II) analysis of the AMI subgroup revealed no statistically significant difference in EMPs and RMPs levels between the STEMI and no-STEMI patients; (III) the levels of EMPs and RMPs in peripheral blood in the multivessel coronary artery disease group were significantly higher than the single-vessel disease group, suggesting that EMPs and RMPs may be involved in AMI and may be correlated with several coronary artery diseases; (IV) the results of our study showed that EMPs levels in the high-risk group and the low-risk group were associated with the highest TIMI risk scores, but no difference in RMPs levels were found among the three groups; (V) last, we examined the diagnostic value of EMPs and RMPs in discriminating patients with AMI from patients with no-CAD. Our results showed that the AUC of circulating EMPs and RMPs were 0.706 and 0.668 . This evidence demonstrated that the high levels of EMPs could more likely to be potential biomarkers than RMPs to distinguish patients with AMI from patients with no-CAD.

The present work must be interpreted with considering the following limitations. First, the analysis of EMPs and RMPs levels in the AMI subgroup required to expand sample size to further assess differences. The TIMI score is a risk assessment parameter for the prognosis of patients with ACS. Variables from a TIMI trial population are selected by multivariate logistic regression analysis to select independent variables for prognostic evaluations, which are helpful for judging the clinical prognosis of patients and selecting the best treatment plan. This study analysed only TIMI risk stratification in EMPs and RMPs levels, and no prognostic correlation analysis was performed. The specific mechanism of EMP and RMP involved in acute thrombotic events of AMI is still unclear, so related cell culture and animal experiments must be added. EMPs and RMPs should be dynamically monitored after AMI to further assess whether those can be used as early diagnosis biomarkers of AMI.

\section{Acknowledgments}

Funding: This work was supported by the National Natural 
Science Foundation of China (No.: 81760068). Project of Xinjiang Uygur Autonomous Region People's Hospital (No.: 20190207).

\section{Footnote}

Reporting Checklist: The authors have completed the STARD reporting checklist. Available at http://dx.doi.org/10.21037/ apm-20-397

Data Sharing Statement: Available at http://dx.doi. org/10.21037/apm-20-397

Peer Review File: Available at http://dx.doi.org/10.21037/ apm-20-397

Conflicts of Interest: All authors have completed the ICMJE uniform disclosure form (available at http://dx.doi. org/10.21037/apm-20-397). The authors have no conflicts of interest to declare.

Ethical Statement: The authors are accountable for all aspects of the work in ensuring that questions related to the accuracy or integrity of any part of the work are appropriately investigated and resolved. The trial was conducted in accordance with the Declaration of Helsinki (as revised in 2013). The study was approved by the Ethics Committee of People's Hospital of Xinjiang Uygur Autonomous Region (No. 2017041), and all patients provided a signed informed consent form.

Open Access Statement: This is an Open Access article distributed in accordance with the Creative Commons Attribution-NonCommercial-NoDerivs 4.0 International License (CC BY-NC-ND 4.0), which permits the noncommercial replication and distribution of the article with the strict proviso that no changes or edits are made and the original work is properly cited (including links to both the formal publication through the relevant DOI and the license). See: https://creativecommons.org/licenses/by-nc-nd/4.0/.

\section{References}

1. GBD 2017 Causes of Death Collaborators. Global, regional, and national age-sex-specific mortality for 282 causes of death in 195 countries and territories, 1980-2017: a systematic analysis for the Global Burden of Disease Study 2017. Lancet 2018;392:1736-88.
2. Wang HW, Huang TS, Lo HH, et al. Deficiency of the microRNA-31-microRNA-720 pathway in the plasma and endothelial progenitor cells from patients with coronary artery disease. Arterioscler Thromb Vasc Biol 2014;34:857-69.

3. Bollati V, Angelici L, Rizzo G, et al. Microvesicleassociated microRNA expression is altered upon particulate matter exposure in healthy workers and in A549 cells. J Appl Toxicol 2015;35:59-67.

4. Aljakna A, Fracasso T, Sabatasso S. Molecular tissue changes in early myocardial ischemia: from pathophysiology to the identification of new diagnostic markers. Int J Legal Med 2018;132:425-38.

5. Xu R, Greening DW, Zhu HJ, et al. Extracellular vesicle isolation and characterization: toward clinical application. J Clin Invest 2016;126:1152-62.

6. Nomura S, Shimizu M. Clinical significance of procoagulant microparticles. J Intensive Care 2015;3:2.

7. VanWijk MJ, VanBavel E, Sturk A, et al. Microparticles in cardiovascular diseases. Cardiovasc Res 2003;59:277-87.

8. Suades R, Padró T, Alonso R, et al. High levels of TSP1+/ $\mathrm{CD} 142+$ platelet-derived microparticles characterise young patients with high cardiovascular risk and subclinical atherosclerosis. Thromb Haemost 2015;114:1310-21.

9. Suades R, Padró T, Badimon L. The Role of Blood-Borne Microparticles in Inflammation and Hemostasis. Semin Thromb Hemost 2015;41:590-606.

10. Suades R, Padró T, Crespo J, et al. Circulating microparticle signature in coronary and peripheral blood of ST elevation myocardial infarction patients in relation to pain-to-PCI elapsed time. Int J Cardiol 2016;202:378-87.

11. Suades R, Padró T, Vilahur G, et al. Growing thrombi release increased levels of $\mathrm{CD} 235 \mathrm{a}(+)$ microparticles and decreased levels of activated platelet-derived microparticles. Validation in ST-elevation myocardial infarction patients. J Thromb Haemost 2015;13:1776-86.

12. Abbas M, Jesel L, Auger C, et al. Endothelial Microparticles From Acute Coronary Syndrome Patients Induce Premature Coronary Artery Endothelial Cell Aging and Thrombogenicity: Role of the Ang II/AT1 Receptor/ NADPH Oxidase-Mediated Activation of MAPKs and PI3-Kinase Pathways. Circulation 2017;135:280-96.

13. Leroyer AS, Isobe H, Lesèche G, et al. Cellular origins and thrombogenic activity of microparticles isolated from human atherosclerotic plaques. J Am Coll Cardiol 2007;49:772-7.

14. Nishiguchi T, Imanishi T, Akasaka T, et al. microRNAs 
and Cardiovascular Diseases. BioMed Research International 2015;2015:682857.

15. Ma S, Tian XY, Zhang Y, et al. E-selectin-targeting delivery of microRNAs by microparticles ameliorates endothelial inflammation and atherosclerosis. Sci Rep 2016;6:22910.

16. Mallat Z, Benamer H, Hugel B, et al. Elevated levels of shed membrane microparticles with procoagulant potential in the peripheral circulating blood of patients with acute coronary syndromes. Circulation 2000;101:841-3.

17. Cheng F, Wang Y, Li J, et al. Berberine improves endothelial function by reducing endothelial microparticles-mediated oxidative stress in humans. Int J Cardiol 2013;167:936-42.

18. Tual-Chalot S, Gagnadoux F, Trzepizur W, et al. Circulating microparticles from obstructive sleep apnea syndrome patients induce endothelin-mediated angiogenesis. Biochim Biophys Acta 2014;1842:202-7.

19. Edrissi H, Schock SC, Hakim AM, et al. Microparticles

Cite this article as: Yuan Y, Maitusong M, Muyesai N. Association of endothelial and red blood cell microparticles with acute myocardial infarction in Chinese: a retrospective study. Ann Palliat Med 2020;9(4):1564-1570. doi: 10.21037/ apm-20-397 generated during chronic cerebral ischemia increase the permeability of microvascular endothelial barriers in vitro. Brain Res 2016;1634:83-93.

20. Hromada C, Mühleder S, Grillari J, et al. Endothelial Extracellular Vesicles-Promises and Challenges. Front Physiol 2017;8:275.

21. Nijiati M, Saidaming A, Guoqing L. In vitro Study of the Thrombogenic Activity of Platelet-derived Microparticles from Patients with Acute Coronary Syndrome. Ann Clin Lab Sci 2017;47:156-61.

22. Nijiati M, Gao Y, Abudureheman Z, et al. Relationship between the Level of Circulating Endothelial MicroParticles in the Blood and Blood Lipid Content in Uyghur and Han Patients with Acute Coronary Syndrome. Clin Lab 2015;61:1071-5.

23. Yuan Y, Muyesai N, Abdeyrim A. Comparison of erythrocyte microparticle levels between acute coronary syndrome and stable angina pectoris patients. Int J Clin Exp Med 2018;11:9528-35. 